\title{
An Image Retrieval Method Based on PGH and Distance Histogram
}

\author{
X. Zou, F.C. You \\ College of Information Engineering \\ Beijing Institute of Graphic Communication \\ Beijing, China
}

\begin{abstract}
An image retrieval method based on contour feature is proposed in this work. Pair-wise Geometric Histogram(PGH) captures the outer contour feature of the target shape. Distance histogram describes the interior details. Combining with two features, a twice retrieval strategy is proposed. Firstly, the query image and database images are compared with PGH. The eligible images are as candidates. Then, the secondary retrieval is carried out between the query image and candidate images. Images meeting the conditions are as the final result. Experiments show that the proposed method can resist rotation, translation transformation and scaling transformation, which has a good performance.
\end{abstract}

\section{Keywords- image retrieval; PGH; distance histogram}

\section{INTRODUCTION}

Content-based Image Retrieval(CBIR) is that, the visual characteristics of the content of the image are extracted, and these characteristics are measured in certain rules to retrieve similar images. The commonly used characteristics are: color, texture, shape, etc. Because humans tend to distinguish the object according to the shape of the target, shape features have attracted much attention. Representation and description of the shape are the core issue of image retrieval based on shape. Existing shape description methods can be divided into two broad categories based on the contour and area. The first category is based on the use of the contour information of the shape of the outer edge. It is consistent with human habit of identifying objects based on edge. The main methods include Fourier descriptors, wavelet descriptors, curvature scale space, etc. The second class uses the entire region information of shapes, and is insensitive to noise and slight deformation. Feature extraction is relatively simple. The main methods include invariant moments, Zernike moments, generalized Fourier descriptors, etc[1,2,3].

Many documents and human visual perception show that the shape features description of combination has better performance than a single feature. Therefore, this paper puts forward a method combining outer contour features with internal details features. Two features respectively choose PGH and distance histogram. Experiments are carried out on standard database, showing that the proposed method is better than single feature retrieval.

\section{II.PAIR-WISE GEOMETRIC HISTOGRAM}

\section{A. Evans' $P G H$}

PGH is a shape descriptor,proposed for the first timeby Evans in 1993. It can be applied also to an irregular shape if the shape is first approximated with a polygon. PGH describes a shape with a relative angle and perpendicular distances $[4,6]$. The angle is the one between two line segments or its extension cord.The perpendicular distance is the one from the endpoint of a line segment to the other line segment or its extension if these two segments are not parallel. As shown in fig.1, The following strategy can be used to calculate PGH. Let each line segment be a reference line on its turn. Then the relative angle $\theta \in[0, \pi]$ and the perpendicular minimum and maximum distances $\left(\mathrm{d}_{\min }\right.$ and $\left.\mathrm{d}_{\max }\right)$ are calculated between the reference line and all the other lines. PGH is a two-dimensional histogram, and its two dimensions are angle and distance. The histogram values are increased by one on the indexes corresponding to the angle $\theta$ and the line segment from the $d_{\min }$ to $d_{\max }[8]$.



(a) 4

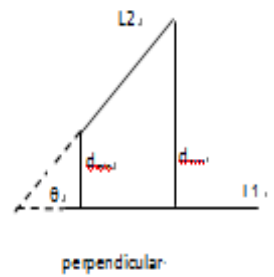

(b) +
FIGURE I. PGH GRAPH.

Using a pair of geometric features to describe the shape of image reduces the generation of uncertainty by only using one feature. The relative angle and the perpendicular distances remain unchanged when translation and rotation occur. PGH has translational and rotational invariance, but without scale invariance. For the shortcoming, there is a need to improve it. Obviously, this problem is only related to the distance, does not involve the angle. So the distances needto be normalized. First of all, the largest one of all $d_{\max }$ is chosen as $d_{\text {norm }}$; Then, a series of normalized distances are got by $\mathrm{d}_{\min } / \mathrm{d}_{\text {norm }}$ and $\mathrm{d}_{\max } / \mathrm{d}_{\text {norm }}$.

\section{B. Pgh Feature Extraction}

The graphic shape may not be a regular polygon. If it has curved edges, each curved edge will be split into many small 
segments, which will not only increase the amount of computation, but also decrease the accuracy. To avoid aboveproblems, preprocessing is a necessary step. Calculating the convex hull of the shape is a good choice, as shown in fig. 2 .

Fig. 2 shows three original images, contours and convex hulls. fig.2(a) is the original images, fig.2(b) is contours, and fig.2(c) is the convex hulls. Asseen in the fig.2: The second one has the scale and rotation transformations compared with the first image, and both of them have similar contours and convex hulls; The contour of the third image is quite different from the former two, but has a similar convex hull. Therefore, PGHs of the three images are highly similar, which will be verified by calculating similarity in the latter stage.
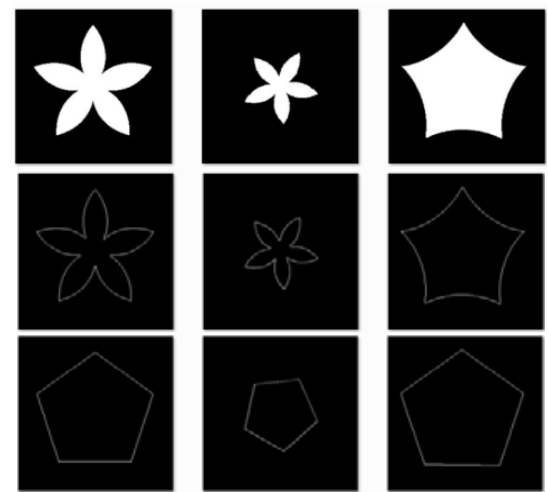

\section{(b)}

FIGURE II. (A) ORIGINAL IMAGES, (B) CONTOURS, AND (C) CONVEX HULLS.

Processing the given image into a convex hull imageensures the validity of the feature, which has itsadvantage. But ignoring the interior details of shape will lead to that unlike images are among the retrieval results. The distance histogram to be mentioned will compensate for this defect perfectly.

\section{DISTANCE HISTOGRAM}

Distancehistogram is definedas to calculate the distance between contour points with its center of mass and quantize the distance to a certain range. The number of contour points which are quantified to each certain range is $d_{i}\left(i=0,1, \ldots, d_{L-1}\right)$ and the quantitative level is $\mathrm{L}$, so the distance histogram can be described as :

$$
\mathrm{D}=\left(\mathrm{d}_{0}, \mathrm{~d}_{1}, \ldots \mathrm{d}_{\mathrm{L}-1}\right)
$$

The distance histogram contains the information of the shape characteristics that reflect the statistical characteristics of the distribution of contour points, so it can be used to describe the shape of the target object. Obviously, distance histogram has translation, rotation invariance, and also has the scale invariance after the normalized processing $[5,7]$.

The fig. 3 shows the distance histograms of three images. The horizontal axis represents quantitative level, and the vertical axis represents the number of the corresponding contour points(the maximum value is normalized to 1). fig.3(b) has rotation and scale transform compared with fig.3(a), but their distance histograms are very similar. fig.3(c) is different with the former two images and its distance histogram is also different with those of the former two images, which proves that the feature of distance histogram can effectively distinguish these images.

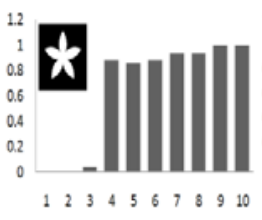

(a)

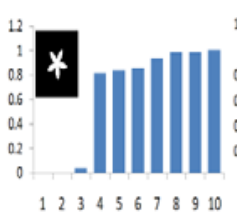

(b)

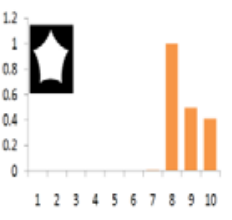

(c)
FIGURE III. DISTANCE HISTOGRAM.

However, distance histogram only reflects the statistical distribution characteristics of the distances between contour points and centroid, but does not reflect the space distribution characteristics of contours points. Therefore two different shapes may have the same distance histogram. So the performance of image retrieval will be unsatisfactory if only using distance histogramas the feature descriptor of image retrieval. The distance histogram is more suitable as an auxiliary feature.

\section{CALCULATION OF SIMILARITY}

There are four methods to calculate histogram similarity.

(1) Relevance

$$
d_{\text {correl }}\left(H_{1}, H_{2}\right)=\frac{\sum_{i} H_{1}^{\prime}(i), ? H_{2}^{\prime}(i)}{\sqrt{\sum_{i} H_{1}^{\prime 2}(i), ? H_{2}^{\prime 2}(i)}}
$$

Among the formula , the $\mathrm{N}$ is the number of the bin of the histogram.

For the relevance matching, the higher the degree of the matching is, the larger the value is. The value 1 is an exact match. The value of a complete mismatch is -1 . A value of 0 indicates no correlation(random combinations).

(2) Chi-square coefficient

$$
d_{\text {chi-square }}\left(H_{1}, H_{2}\right)=\sum_{i} \frac{\left(H_{1}(i)-H_{2}(i)\right)^{2}}{H_{1}(i)+H_{2}(i)}
$$

For, the matching degree of low grade is higher than high grade. A value of 0 indicates exact match. The value of a complete mismatch is infinite (depending on the size of the histogram).

(3) The correlation coefficient

$$
d_{\text {intersection }}\left(H_{1}-H_{2}\right)=\sum_{i} \min \left(H_{1}(i), H_{2}(i)\right)
$$

For histogram intersection, the high grade marks a good 
match. Of course, the low grade marks a bad match. The grade 1 marks exact match and the 0 marks mismatch if the two histograms are normalized to 1 .

(4) Bhattacharyya distance

$$
d_{\text {Bhattacharyya }}\left(H_{1}, H_{2}\right)=\sqrt[?]{-\sum_{i} \frac{\sqrt{H_{1}(i) \cdot H_{2}(i)}}{\sum_{i} H_{1}(i) \cdot \sum_{i} H_{2}(i)}}
$$

For Bhattacharyya distance, the low grade marks a good match and the high grade marks a bad match. The 0 means exact match and the 1 means complete mismatch.

In general, we should normalize histograms before comparing them. The concepts such as histogram intersection have no meaning if histograms have not been normalized (even if allow). Experience has shown that in the case of fast but not very accurate matching, the effect of histogram intersection method is better and in the case of a slow but more precise matching, the effect of using chi-square distance orBhattacharyya distance is better.

\section{IMAGE RETRIEVAL EXPERIMENTS BASED ON PGH AND DISTANCE HISTOGRAM}

The strategy adopts twice retrievals. Bhattacharyya distance is applied to calculate similarity.PGH method is used in the first round. The query image and each image in the database match in turn. Images from the databaseare marked as candidates, whose distances with the query image are less than a given threshold value. The second round of retrieval uses distance histogram. The condition is similar. Distances between the query image and the candidates are calculated. If the distance is less than the given threshold value, the corresponding image is selected.

In order to verify the validity of the proposed method, part of the MPEG-7 image testing library is as the image database. But the images of MPEG-7 are not deliberately designed for performance evaluation of CBIR systems. For instance, most of these images are usually semantically and perceptually alike but not structurally and conceptually alike.However, the greatest value of a retrieval method is to locate the images that are highly homogeneous, rotated and scaled to the query example. Therefore we create an image dataset based on MPEG-7 for testing the algorithms against structural changes of images.Some images of the image dataset are shown in fig.4.

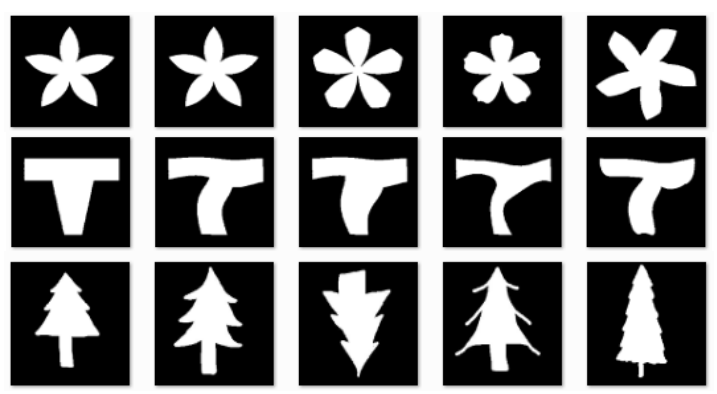

FIGURE IV. SOME IMAGES IN RETRIEVAL IMAGES LIBRARY.
The precision-recall graph is commonly used for evaluating the performance of an algorithm. The horizontalaxis and verticalaxis respectively represent recall and precision rates. Precision is the ratio of the number of relevant images retrieved to the total number of images retrieved while recall is the ratio of the number of relevant images retrieved to the total number of relevant images stored in the database. The higher the values of precision and recall are, the better the performance of algorithm is. The fig. 5 shows the accuracy-recall rate curves which use the method proposed in this paper and the single PGH algorithm. We can see that the algorithm proposed in paper is better than single PGH algorithm.

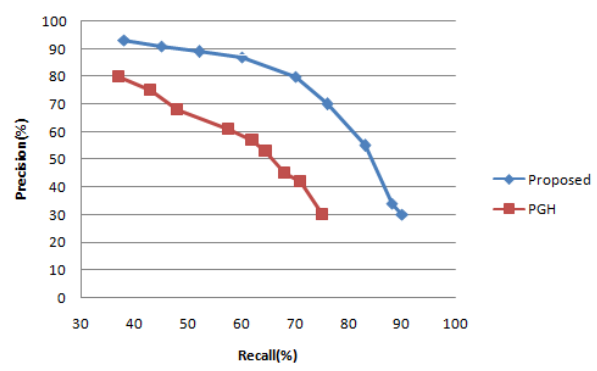

FIGURE V. PRECISION-RECALL GRAPH.

\section{CONCLUSION}

Shape is often used as the basis of image retrieval, because itis the most intuitive feature in the underlying image characteristics. However, the two kinds of character description that come from external shape contour and internal structure may have very big differences. The combined method is proposed in this paper and the experimental results show that is better than using single feature in image retrieval. However, it still needs future research for the images with complex shapeor the images withsimilar semantic and different structural shape. With the development of image retrieval technology, combining the low-level visual features with the high-level semantic will be a new development trend.

\section{ACKNOWLEDGEMENTS}

This paper is supported by Scientific Research Key Project of Beijing Municipal Commission of Education (KZ201210015015)and Beijing Natural Science Foundation (4122026).

\section{REFERENCES}

[1] D.S. Zhang, G.J. Lu, Review of Shape Representation and Description Techniques.Pattern Recognition,37:pp. 1-19, 2004.

[2] Q. Qiu, Shape descriptor construction and its application in trademark image retrieval[D].HuNan: Xiangtan University, 2013.

[3] J.W. Chen, X.Y. Wang, J.Yu, Edge-based Color Image Retrieval by Using Multiple Histograms[J]. Journal of Chinese Computer Systems, 05 : pp. 978-983, 2010.

[4] X.D. Yang, L.D. Wu, Y.X. Xie, Z. Yang, W. Zhou, Local description and retrieval method for binary image contour[J]. Journal of Computer Applications,01:pp. 65-67, 2010.

[5] J.X. Zeng, Y.G. Zhao, X. Fu, An Improved Distance Coherence Vector Algorithm for CBIR[J]. Pattern Recognition and Artificial Intelligence05: pp. 715-719, 2010.

[6] Q.Y. Yang, Research on Image Retrieval Method of Combining SIFT Feature with PGH[D].Chongqing University,2013. 
[7] L. Guo, X.H. Sun, Y.Y. Huang, J.Y. Yang, Distance Distribution Histogram and its Application in Trademark Image Retrieval[J]. Journal of Image and Graphics, 10: pp. 45-49, 2002.

[8] M.Z. Wu, Improvement of Contour-Based Image Retrieval using Pair-wise Geometric Histogram[D].Jinan University,2006. 